\title{
A CLASS OF EXTREMAL FUNCTIONS FOR THE FOURIER TRANSFORM
}

BY

\author{
S. W. GRAHAM AND JEFFREY D. VAALER ${ }^{1}$
}

\begin{abstract}
We determine a class of real valued, integrable functions $f(x)$ and corresponding functions $M_{f}(x)$ such that $f(x)<M_{f}(x)$ for all $x$, the Fourier transform $\hat{M}_{f}(t)$ is zero when $|t|>1$, and the value of $\hat{M}_{f}(0)$ is minimized. Several applications of these functions to number theory and analysis are given.
\end{abstract}

1. Introduction. Let $f(x)$ be a bounded, real valued, integrable function. This paper will be concerned with the problem of finding an integrable real valued function $G(x)=G_{f}(x)$ such that

$$
\begin{aligned}
& f(x) \leqslant G(x) \text { for all real } x, \text { and } \\
& \hat{G}(t)=\int_{-\infty}^{\infty} G(x) e^{-2 \pi i t x} d x=0 \text { if }|t| \geqslant 1 .
\end{aligned}
$$

If $G$ satisfies (1.1) and (1.2) then we say that $G$ is a majorant of $f$. If $\hat{G}(0)$ is minimal for all functions satisfying (1.1) and (1.2), we say that $G$ is an extreme majorant of $f$.

Our work is motivated by the following results of Beurling and Selberg. (For proofs of these results see $\$ 7$ of Montgomery [5].) Let $z=x+i y$ and

$$
\operatorname{sgn}(x)= \begin{cases}x|x|^{-1} & \text { if } x \neq 0 \\ 0 & \text { if } x=0\end{cases}
$$

Beurling observed that if

$$
F(z)=\left(\frac{\sin \pi z}{\pi}\right)^{2}\left\{\sum_{n=0}^{\infty}(z-n)^{-2}-\sum_{n=1}^{\infty}(z+n)^{-2}+2 z^{-1}\right\},
$$

then $F(x) \geqslant \operatorname{sgn}(x)$ for all real $x$ and

$$
\int_{-\infty}^{\infty} F(x)-\operatorname{sgn}(x) d x=1
$$

$F$ is not integrable on $\mathbf{R}$, but $F(z)=O\left(e^{2 \pi|y|}\right)$, and this can be interpreted to mean $\hat{F}(t)=0$ for $|t| \geqslant 1$. Among all such functions which majorize $\operatorname{sgn}(x), F$ is the unique function which minimizes the quantity in (1.3). Selberg noted that if $l>0$ and

$$
G_{l}(x)=\frac{1}{2}\{F(x)+F(l-x)\}
$$

Received by the editors May 5, 1980.

1980 Mathematics Subject Classification. Primary 42A68; Secondary 10H30, 40E05.

${ }^{1}$ The research of the second author was supported by the National Science Foundation, grant number MCS77-01830. 
then $G_{l}$ is a majorant of $\chi_{[0, l]}$, the characteristic function of $[0, l]$. If $l$ is an integer then $G_{l}$ is an extreme majorant of $\chi_{[0, l]}$.

Here we will consider the problem of constructing extreme majorants for functions $f$ satisfying

$f$ is a bounded, real valued, integrable function,

$f$ is continuously differentiable at all $x \neq 0$,

$f$ has both left- and right-hand limits at $x=0$ with $f(0)=\lim _{x \rightarrow 0} \sup f(x)$, and

$$
\begin{aligned}
& \sum_{n \neq 0}\left\{|f(x+n)|+\left|f^{\prime}(x+n)\right| \log 2|n|\right\} \text { is uniformly conver- } \\
& \text { gent for }|x| \leqslant \frac{1}{2} .
\end{aligned}
$$

Although these conditions can be modified in various ways, it is essential that $f$ have at most one discontinuity; see the comments at the end of $\$ 2$. Since $f$ is to be majorized by a continuous function, the value of $f(0)$ is unimportant as long as $f(0) \leqslant \lim \sup _{x \rightarrow 0} f(x)$. For our purposes, however, it is convenient for $f$ to be upper semicontinuous. The condition (1.8) is convenient for technical reasons; it may be possible to weaken this condition and still obtain our results.

In order to describe our method for determining extreme majorants of functions $f(x)$ satisfying (1.5)-(1.8) we make the following observations. The periodic function

$$
P(x)=\sum_{n=-\infty}^{\infty} f(x+n)
$$

is continuous for all $x$ in $[-1 / 2,1 / 2]$ except possibly for a jump discontinuity at $x=0$. If $G$ is a majorant of $f$ then by Lemma 4 (a variant of the Poisson summation formula),

$$
\begin{aligned}
P(x) & =\lim _{N \rightarrow \infty} \sum_{n=-N}^{N}\left(1.13-\frac{|n|}{N}\right) f(x+n) \\
& \leqslant \lim _{N \rightarrow \infty} \sum_{n=-N}^{N}\left(1-\frac{|n|}{N}\right) G(x+n)=\hat{G}(0)
\end{aligned}
$$

for all $x$. Since $P(x)$ is upper semicontinuous, $\sup _{|x|<1 / 2} P(x)=P\left(x_{0}\right)$ for some point $x_{0}$ in $[-1 / 2,1 / 2)$. If $P\left(x_{0}\right)=\hat{G}(0)$ then $G$ is clearly an extreme majorant of $f$. We therefore consider two cases.

First assume that $0<\left|x_{0}\right| \leqslant 1 / 2$. Then a natural choice for an extreme majorant is

$$
\begin{aligned}
M_{f}(x)= & \left(\frac{\sin \pi\left(x-x_{0}\right)}{\pi}\right)^{2} \\
& \cdot\left\{\sum_{n=-\infty}^{\infty} f\left(x_{0}+n\right)\left(x-x_{0}-n\right)^{-2}+\sum_{n=-\infty}^{\infty} f^{\prime}\left(x_{0}+n\right)\left(x-x_{0}-n\right)^{-1}\right\} .
\end{aligned}
$$


Note that $M_{f}\left(x_{0}+n\right)=f\left(x_{0}+n\right)$ and $M_{f}^{\prime}\left(x_{0}+n\right)=f^{\prime}\left(x_{0}+n\right)$ for all integers $n$. Also, since $P(x)$ has a local maximum at $x_{0}$,

$$
P^{\prime}\left(x_{0}\right)=\sum_{n=-\infty}^{\infty} f^{\prime}\left(x_{0}+n\right)=0 .
$$

(That the sum can be differentiated term by term follows from (1.8).) In Lemma 4 we show that $(1.12)$ is a necessary condition for $M_{f}(x)$ to be integrable.

If $x_{0}=0$ then our choice for an extreme majorant is

$$
M_{f}(x)=\left(\frac{\sin \pi x}{\pi}\right)^{2}\left\{\sum_{n=-\infty}^{\infty} f(n)(x-n)^{-2}+\sum_{n \neq 0} f^{\prime}(n)(x-n)^{-1}-x^{-1} \sum_{n \neq 0} f^{\prime}(n)\right\} .
$$

Again we find that $M_{f}(n)=f(n)$ for all integers $n, M_{f}^{\prime}(n)=f^{\prime}(n)$ if $n \neq 0$, and

$$
\sum_{n=-\infty}^{\infty} M_{f}^{\prime}(n)=0 \text {. }
$$

If $P(x)$ takes its maximum value at several points in the interval $[-1 / 2,1 / 2)$ then we can construct $M_{f}$ for each such point. In $\$ 2$ we will show that if $P(x)$ takes its maximum value at $x_{0}$ and the corresponding function $M_{f}$ is a majorant of $f$ then it is the unique extreme majorant.

We also consider minorizing functions. We say that $g$ is a minorant of $f$ if

$$
\begin{aligned}
& g(x) \leqslant f(x) \text { for all real } x, \text { and } \\
& \hat{g}(t)=0 \text { for all }|t|>1 .
\end{aligned}
$$

If $\hat{g}(0)$ is maximal for all functions satisfying (1.14) and (1.15), we say that $g$ is an extreme minorant of $f$. Clearly $g$ is a minorant (extreme minorant) of $f$ if and only if $-g$ is a majorant (extreme majorant) of $-f$. Thus all the results which we prove for extreme majorants have obvious analogues for extreme minorants. If $-f$ satisfies (1.5)-(1.8) and

$$
-\sum_{n=-\infty}^{\infty} f(x+n)
$$

takes its maximum value at $x_{0}$, then we write $m_{f}(x)=-M_{-f}(x)$.

Our main problem, therefore, is to determine which functions $f$ satisfy

$$
m_{f}(x) \leqslant f(x) \leqslant M_{f}(x)
$$

for all real $x$. We solve this problem only for certain special functions. Let $\lambda$ be a positive parameter and define

$$
E(\lambda, x)= \begin{cases}e^{-\lambda x} & \text { if } x>0 \\ 0 & \text { if } x<0\end{cases}
$$

In $\$ 3$ we show that (1.16) holds for the functions $E(\lambda, x), \operatorname{sgn}(x) e^{-\lambda|x|}$, and $e^{-\lambda|x|}$. We also prove that if $\nu$ is a finite Borel measure on $(0, \infty)$ such that

$$
\int_{0}^{\infty} \lambda^{-1} d \nu(\lambda)<\infty
$$


then (1.16) is satisfied for any function $f$ of the form

$$
\begin{aligned}
& f(x)=\int_{0}^{\infty} E(\lambda, x) d \nu(\lambda), \\
& f(x)=\int_{0}^{\infty} \operatorname{sgn}(x) e^{-\lambda|x|} d \nu(\lambda),
\end{aligned}
$$

or

$$
f(x)=\int_{0}^{\infty} e^{-\lambda|x|} d \nu(\lambda) .
$$

In each case the periodic function (1.9) has a unique maximum on $[-1 / 2,1 / 2)$, and so there is only one function $M_{f}$ which is a possible extreme majorant. Similarly, there is only one function $m_{f}$ which is a possible extreme minorant.

We remark that the conditions (1.5)-(1.8) are not sufficient to ensure that $M_{f}(x)$ is a majorant of $f$. A simple example is provided by the function

$$
h(x)= \begin{cases}-6 x^{5}+15 x^{4}-10 x^{3}+1 & \text { if } 0 \leqslant x \leqslant 1, \\ 0 & \text { if } x<0 \text { or } 1<x .\end{cases}
$$

The periodic function $\sum_{n=-\infty}^{\infty} h(x+n)$ has a unique maximum on $[-1 / 2,1 / 2)$ at $x=0$, and so from $(1.13)$ we find that $M_{h}(x)=(\sin \pi x / \pi x)^{2}$. But the inequality $h(x) \leqslant M_{h}(x)$ does not hold for small positive values of $x$, since $M_{h}^{\prime}(0+)=$ $h^{\prime}(0+)=0$ and $M_{h}^{\prime \prime}(0+)<0=h^{\prime \prime}(0+)$.

In $\$ 4$ we give a sharp version of the Wiener-Ikehara theorem; the proof makes use of the extreme majorants and extreme minorants of $E(\lambda, x)$. In the final section we give some other applications to number theory and analysis.

Notation. As we noted in (1.2), we define our Fourier transforms using $e^{-2 \pi i t x}$; therefore it is convenient to use the notation $e(x)=e^{2 \pi i x}$. The letter $x$ is reserved for a real variable, $n$ denotes an integer variable, and $z=x+i y$ denotes a complex variable. We use the Landau notation $O$ and Vinogradov's notation «; $f \ll g$ means $f=O(g)$. All constants implied by " $O$ " and “ $\ll$ " are absolute unless dependence is indicated by a subscript. We usually use $[a, b]$ to denote the closed interval from $a$ to $b$, although in $\$ 5$ we also use $[a, b]$ to denote the least common multiple of $a$ and $b$. The precise meaning should be clear from the context. In $\$ 5$ we use $\varphi$ and $\mu$ to denote the number-theoretic functions of Euler and Möbius, respectively.

2. Uniqueness of extremal functions. Throughout this section we assume that $f(x)$ satisfies the conditions (1.5)-(1.8) and we let

$$
P(x)=\sum_{n=-\infty}^{\infty} f(x+n) .
$$

We state and prove our results only for majorants. If $-f$ satisfies (1.5)-(1.8) then these results can be applied to $m_{f}(x)=-M_{-f}(x)$.

THeOREM 1. Suppose that $P(x)$ takes it maximum value at $x_{0} \in[-1 / 2,1 / 2)$. If the corresponding function $M_{f}$ is a majorant of $f$ then it is the unique extreme majorant.

The proof of Theorem 1 rests upon the following technical lemmata. 
LEMMA 2. If $n \neq 0$ is an integer and $t$ is real, then

$$
\sum_{r=-\infty}^{\infty}\left|\frac{\sin \pi t}{r+t-n}-\frac{\sin \pi t}{r+t}\right| \ll \log 2|n| .
$$

Proof. By periodicity, we may assume that $|t| \leqslant 1 / 2$. The terms with $r=0$ and $r=n$ contribute $O(1)$ to the sum. All other terms are

$$
\ll\left|(r-n)^{-1}-r^{-1}\right|
$$

so it suffices to bound

$$
\sum_{\substack{r=-\infty \\ r \neq 0, r \neq n}}^{\infty}\left|(r-n)^{-1}-r^{-1}\right|
$$

Since (2.1) is even in $n$, we may suppose that $n$ is positive. Also, the term with index $r$ is equal to the term with index $n-r$, so (2.1) is

$$
\begin{aligned}
& \ll \sum_{\substack{r=1 \\
r \neq n}}^{2 n}\left|(r-n)^{-1}-r^{-1}\right|+\sum_{r=2 n}^{\infty} \frac{n}{r(r-n)} \\
& \ll \log 2 n+n \sum_{r=2 n}^{\infty} r^{-2} \ll \log 2 n .
\end{aligned}
$$

LEMMA 3. If $M_{f}(x)$ is defined by (1.13) then

$$
\int_{-\infty}^{\infty}\left|M_{f}(x)\right| d x \ll|f(0)|+\sum_{n \neq 0}\left\{|f(n)|+\left|f^{\prime}(n)\right| \log 2|n|\right\} .
$$

Proof. For any integer $n$,

$$
\int_{-\infty}^{\infty}\left(\frac{\sin \pi t}{\pi(t-n)}\right)^{2} d t=1
$$

and for $n \neq 0$,

$$
\int_{-\infty}^{\infty}\left|\frac{\sin \pi t}{t-n}-\frac{\sin \pi t}{t}\right| d t=\sum_{r=-\infty}^{\infty} \int_{-1 / 2}^{1 / 2}\left|\frac{\sin \pi t}{r+t-n}-\frac{\sin \pi t}{r+t}\right| d t \ll \log 2|n|
$$

by Lemma 2 . Therefore

$$
\int_{-\infty}^{\infty}\left(\frac{\sin \pi t}{\pi}\right)^{2}\left|\sum_{n=-\infty}^{\infty} f(n)(t-n)^{-2}\right| d t \leqslant \sum_{n=-\infty}^{\infty}|f(n)|
$$

and

$$
\begin{aligned}
& \int_{-\infty}^{\infty}\left(\frac{\sin \pi t}{\pi}\right)^{2}\left|\sum_{n \neq 0} f^{\prime}(n)(t-n)^{-1}-t^{-1} \sum_{n \neq 0} f^{\prime}(n)\right| d t \\
& \quad \leqslant \int_{-\infty}^{\infty}\left(\frac{\sin \pi t}{\pi}\right)^{2} \sum_{n \neq 0}\left|f^{\prime}(n)\right|\left|(t-n)^{-1}-t^{-1}\right| d t \\
& \ll \sum_{n \neq 0}\left|f^{\prime}(n)\right| \log 2|n| .
\end{aligned}
$$


LeMmA 4. Let $G(z)$ be an entire function such that $G$ is integrable on the real line and $\hat{G}(t)=0$ if $|t| \geqslant 1$. Then for all real $x$,

$$
\lim _{N \rightarrow \infty} \sum_{n=-N}^{N}\left(1-\frac{|n|}{N}\right) G(x+n)=\hat{G}(0)
$$

and

$$
\lim _{N \rightarrow \infty} \sum_{n=-N}^{N}\left(1-\frac{|n|}{N}\right) G^{\prime}(x+n)=0 .
$$

Furthermore, if there exists a number $x_{0}$ such that $G\left(x_{0}+n\right)=G^{\prime}\left(x_{0}+n\right)=0$ for every integer $n$, then $G(z)=0$ for all complex $z$.

Proof. It suffices to prove (2.2) and (2.3) with $x=0$ since the translate $G(z-x)$ satisfies all the hypotheses of the lemma. Similarly, in proving the last assertion of the lemma we may suppose that $x_{0}=0$.

For $0 \leqslant t \leqslant 1$ define

$$
u(t)=\hat{G}(t)+\hat{G}(t-1)
$$

and

$$
v(t)=2 \pi i\{t \hat{G}(t)+(t-1) \hat{G}(t-1)\} .
$$

Since $G$ is integrable on the real axis, $\hat{G}$ is continuous, and so both $u$ and $v$ are continuous on $[0,1]$. Moreover, $u(0)=u(1)=\hat{G}(0)$ and $v(0)=v(1)=0$. Thus $u$ and $v$ may be extended to continuous functions of period 1. Clearly

$$
\hat{G}(t)=(1-|t|) u(t)+(2 \pi i)^{-1} \operatorname{sgn}(t) v(t)
$$

for $|t| \leqslant 1$. For all complex $z$,

$$
G(z)=\int_{-1}^{1} \hat{G}(t) e(t z) d t
$$

and

$$
G^{\prime}(z)=2 \pi i \int_{-1}^{1} t \hat{G}(t) e(t z) d t
$$

Therefore

$$
G(n)=\int_{0}^{1}\{\hat{G}(t)+\hat{G}(t-1)\} e(t n) d t=\int_{0}^{1} u(t) e(t n) d t
$$

and

$$
G^{\prime}(n)=2 \pi i \int_{0}^{1}\{t \hat{G}(t)+(t-1) \hat{G}(t-1)\} e(t n) d t=\int_{0}^{1} v(t) e(t n) d t
$$

for every integer $n$. In other words, $G(n)$ and $G^{\prime}(n)$ are the Fourier coefficients of $u$ and $v$ respectively. Since $u$ and $v$ are continuous,

$$
u(t)=\lim _{N \rightarrow \infty} \sum_{n=-N}^{N}\left(1-\frac{|n|}{N}\right) G(n) e(-t n)
$$

and

$$
v(t)=\lim _{N \rightarrow \infty} \sum_{n=-N}^{N}\left(1-\frac{|n|}{N}\right) G^{\prime}(n) e(-t n) .
$$


Statements (2.2) and (2.3) now follow by taking $t=0$. If $G(n)=G^{\prime}(n)=0$ for all $n$, then $u(t)=v(t)=0$ for all $t, \hat{G}(t)=0$ by (2.4), and so $G(z)=0$ by (2.5).

We now prove Theorem 1 . We suppose that $x_{0}=0$ and hence that $M_{f}$ is defined by (1.13); only trivial modifications are needed if $x_{0} \neq 0$. By Lemma $3, M_{f}$ is integrable. Thus we can compute its Fourier transform from the identities

$$
\int_{-1}^{1}(1-|t|) e(t x) d t=\left(\frac{\sin \pi x}{\pi x}\right)^{2},
$$

and

$$
(2 \pi i)^{-1} \int_{-1}^{1} \operatorname{sgn}(t) e(t x) d t=x^{-1}\left(\frac{\sin \pi x}{\pi}\right)^{2}
$$

We obtain $\hat{M}_{f}(t)=0$ for $|t| \geqslant 1$ and

$$
\begin{aligned}
\hat{M}_{f}(t)= & (1-|t|) \sum_{n=-\infty}^{\infty} f(n) e(-n t) \\
& +(2 \pi i)^{-1} \operatorname{sgn}(t) \sum_{n \neq 0} f^{\prime}(n)\{e(-n t)-1\}
\end{aligned}
$$

for $|t| \leqslant 1$. If $f(x) \leqslant M_{f}(x)$ for all real $x$, then $M_{f}$ is an extreme majorant by (1.10). Now suppose that $G$ is also an extreme majorant. Then

$$
\hat{G}(0)=\hat{M}_{f}(0)=\sum_{n=-\infty}^{\infty} f(n)=P(0),
$$

and so by (2.2), $G(n)=M_{f}(n)=f(n)$ for all $n$. Since $G(x)-f(x)$ has a local minimum at each integer $n, G^{\prime}(n)=f^{\prime}(n)=M_{f}^{\prime}(n)$ for all $n \neq 0$. By (2.3),

$$
G^{\prime}(0)=M_{f}^{\prime}(0)=-\sum_{n \neq 0} f^{\prime}(n)
$$

By Lemma 4, $G(z)=M_{f}(z)$ for all complex $z$.

The fact that $f$ has at most one discontinuity is essential to the uniqueness assertion in Theorem 1. Selberg (unpublished) has shown that if $l$ is a positive integer, then

$$
G(x)=\left(\frac{\sin \pi x}{\pi}\right)^{2}\left\{\sum_{n=0}^{l}(x-n)^{-2}+\beta x^{-1}-\beta(x-l)^{-1}\right\}
$$

is an extreme majorant of $\chi_{[0, l]}$ for any $\beta$ such that

$$
l(l+1)^{-1} \leqslant \beta \leqslant l^{-1}(l+1) .
$$

When $\beta=1, G$ is the function $G_{l}$ defined in (1.4). If $l$ is not an integer then $G_{l}$ is no longer an extreme majorant. B. Logan (unpublished) has shown that the extreme majorant exists and is unique in this case.

3. Majorants and minorants for exponential functions. In this section we determine extreme majorants and extreme minorants for the functions $E(\lambda, x)$ (defined by (1.17)), $\operatorname{sgn}(x) e^{-\lambda|x|}, e^{-\lambda|x|}$, and more generally for the functions $f(x)$ defined by (1.18), (1.19) or (1.20). For $\lambda>0$ and all complex $z$ we set

$$
A(\lambda, z)=\left(\frac{\sin \pi z}{\pi}\right)^{2} \sum_{n=0}^{\infty} e^{-\lambda n}\left\{(z-n)^{-2}-\lambda(z-n)^{-1}\right\}
$$


We also define

$$
B(\omega)= \begin{cases}\omega e^{\omega}\left(e^{\omega}-1\right)^{-1} & \text { for real } \omega \neq 0, \\ 1 & \text { for } \omega=0\end{cases}
$$

LEMMA 5. If $\operatorname{Re}(z)<0$, then

$$
A(\lambda, z)=\left(\frac{\sin \pi z}{\pi}\right)^{2} \int_{0}^{\infty} B(\lambda+\omega) e^{z \omega} d \omega
$$

If $\operatorname{Re}(z)>0$ then

$$
A(\lambda, z)=e^{-\lambda z}-\left(\frac{\sin \pi z}{\pi}\right)^{2} \int_{-\infty}^{0} B(\lambda+\omega) e^{z \omega} d \omega .
$$

Proof. Let $\delta>0$. If $\operatorname{Re}(z) \leqslant-\delta$ then

$$
\int_{0}^{\infty} B(\lambda+\omega) e^{z \omega} d \omega=e^{-\lambda z} \int_{\lambda}^{\infty} B(\omega) e^{z \omega} d \omega=e^{-\lambda z} \int_{\lambda}^{\infty} \omega \sum_{n=0}^{\infty} e^{(z-n) \omega} d \omega .
$$

Now

$$
\left|\omega \sum_{n=0}^{\infty} e^{(z-n) \omega}\right| \leqslant \omega \sum_{n=0}^{\infty} e^{-\delta \omega-n \omega}=B(\omega) e^{-\delta \omega}
$$

so by the dominated convergence theorem

$$
\begin{aligned}
\int_{0}^{\infty} B(\lambda+\omega) e^{z \omega} d \omega & =e^{-\lambda z} \sum_{n=0}^{\infty} \int_{\lambda}^{\infty} \omega e^{(z-n) \omega} d \omega \\
& =\sum_{n=0}^{\infty} e^{-\lambda n}\left\{(z-n)^{-2}-\lambda(z-n)^{-1}\right\}
\end{aligned}
$$

This proves (3.2).

Next suppose that $\operatorname{Re}(z) \geqslant \delta$. Then

$$
\int_{-\infty}^{0} B(\lambda+\omega) e^{z \omega} d \omega=e^{-\lambda z}\left\{\int_{-\infty}^{0} B(\omega) e^{z \omega} d \omega+\int_{0}^{\lambda} B(\omega) e^{z \omega} d \omega\right\}
$$

By the dominated convergence theorem,

$$
\int_{-\infty}^{0} B(\omega) e^{z \omega} d \omega=-\sum_{n=1}^{\infty} \int_{-\infty}^{0} \omega e^{(z+n) \omega} d \omega=\sum_{n=1}^{\infty}(z+n)^{-2}
$$

Furthermore,

$$
\int_{0}^{\lambda} B(\omega) e^{z \omega} d \omega=\sum_{n=0}^{\infty}(z-n)^{-2}-e^{\lambda z} \sum_{n=0}^{\infty} e^{-\lambda n}\left\{(z-n)^{-2}-\lambda(z-n)^{-1}\right\} .
$$

The proof of (3.3) is completed by combining (3.1), (3.4), (3.5) and (3.6).

Since $\sum_{n=-\infty}^{\infty} E(\lambda, x+n)$ has a unique maximum on $[-1 / 2,1 / 2)$ at $x_{0}=0$, the function $M_{E(\lambda, x)}$ is defined by (1.13). For notational convenience we write $M_{E(\lambda, x)}=$ $M(\lambda, x)$. The function $-E(\lambda, x)$ does not satisfy (1.7), since it is not upper semicontinuous. However, if we let

$$
H(\lambda, x)= \begin{cases}E(\lambda, x) & \text { for } x \neq 0 \\ 0 & \text { for } x=0\end{cases}
$$


then $-H(\lambda, x)$ satisfies (1.5)-(1.8) and $-\sum_{n=-\infty}^{\infty} H(\lambda, x+n)$ takes its unique maximum on $[-1 / 2,1 / 2)$ at $x_{0}=0$. Thus we may use $m(\lambda, x)=m_{H(\lambda, x)}(x)=$ $-M_{-H(\lambda, x)}(x)$ to define a minorizing function for $E(\lambda, x)$. We find that

$$
m(\lambda, x)=M(\lambda, x)-\left(\frac{\sin \pi x}{\pi x}\right)^{2}
$$

THEOREM 6. For $\lambda>0$ and all real $x$,

$$
E(\lambda, x)-\left(\frac{\sin \pi x}{\pi x}\right)^{2} \leqslant m(\lambda, x) \leqslant E(\lambda, x) \leqslant M(\lambda, x)<E(\lambda, x)+\left(\frac{\sin \pi x}{\pi x}\right)^{2} .
$$

Proof. First we note that

$$
M(\lambda, x)=A(\lambda, x)+B(\lambda) x^{-1}\left(\frac{\sin \pi x}{\pi}\right)^{2} .
$$

Suppose $x<0$. By Lemma 5,

$$
M(\lambda, x)=\left(\frac{\sin \pi x}{\pi}\right)^{2} \int_{0}^{\infty}\{B(\lambda+\omega)-B(\lambda)\} e^{x \omega} d \omega .
$$

Now $0<B^{\prime}(t)<1$ for all real $t$, so

$$
0<B(\lambda+\omega)-B(\lambda)<\omega
$$

for $\omega>0$. Therefore

$$
0 \leqslant M(\lambda, x) \leqslant\left(\frac{\sin \pi x}{\pi}\right)^{2} \int_{0}^{\infty} \omega e^{x \omega} d \omega=\left(\frac{\sin \pi x}{\pi x}\right)^{2}
$$

for $x<0$.

Suppose $x>0$. By Lemma 5,

$$
M(\lambda, x)-e^{-\lambda x}=\left(\frac{\sin \pi x}{\pi}\right)^{2} \int_{-\infty}^{0}\{B(\lambda)-B(\lambda+\omega)\} e^{x \omega} d \omega .
$$

Since $0 \leqslant B(\lambda)-B(\lambda+\omega)<-\omega$ for $\omega<0$,

$$
0 \leqslant M(\lambda, x)-e^{-\lambda x} \leqslant\left(\frac{\sin \pi x}{\pi}\right)^{2} \int_{0}^{\infty} \omega e^{-x \omega} d \omega=\left(\frac{\sin \pi x}{\pi x}\right)^{2} .
$$

The proof is completed by combining (3.7), (3.8) and (3.9).

We define

$$
M_{1}(\lambda, x)=M(\lambda, x)-m(\lambda,-x)
$$

and

$$
m_{1}(\lambda, x)=m(\lambda, x)-M(\lambda,-x) .
$$

The function $\operatorname{sgn}(x) e^{-\lambda|x|}$ does not satisfy (1.7), but we may use $f(x)=$ $\operatorname{sgn}(x+) e^{-\lambda|x|}$ to determine $M_{f}$ by (1.13). We obtain $M_{f}(x)=M_{1}(\lambda, x)$. Similarly, we find that $m_{1}(\lambda, x)$ is a possible extreme minorant for $\operatorname{sgn}(x) e^{-\lambda|x|}$.

THEOREM 7. For $\lambda>0$ and all real $x$,

$$
\begin{aligned}
\operatorname{sgn}(x) e^{-\lambda|x|}-2\left(\frac{\sin \pi x}{\pi x}\right)^{2} & \leqslant m_{1}(\lambda, x)<\operatorname{sgn}(x) e^{-\lambda|x|}<M_{1}(\lambda, x) \\
& <\operatorname{sgn}(x) e^{-\lambda|x|}+2\left(\frac{\sin \pi x}{\pi x}\right)^{2} .
\end{aligned}
$$


Proof. This follows directly from (3.10), (3.11) and Theorem 6.

If $f(x)=e^{-\lambda|x|}$ then the periodic function defined by (1.9) has a unique maximum on $[-1 / 2,1 / 2)$ at 0 and a unique minimum on $[-1 / 2,1 / 2)$ at $-1 / 2$. A simple computation shows that $M_{f}(x)=M_{2}(\lambda, x)$ and $m_{f}(x)=m_{2}(\lambda, x)$, where

$$
M_{2}(\lambda, x)=A(\lambda, x)+A(\lambda,-x)-\left(\frac{\sin \pi x}{\pi x}\right)^{2}
$$

and

$$
m_{2}(\lambda, x)=e^{-\lambda / 2}\{A(\lambda, x-1 / 2)+A(\lambda,-x-1 / 2)\} .
$$

THEOREM 8. For $\lambda>0$ and all real $x$,

$$
m_{2}(\lambda, x) \leqslant e^{-\lambda|x|}<M_{2}(\lambda, x) .
$$

Proof. All functions in (3.12) are even and continuous, so it suffices to consider $x>0$.

By Lemma 5,

$$
M_{2}(\lambda, x)-e^{-\lambda x}=\left(\frac{\sin \pi x}{\pi}\right)^{2} \int_{0}^{\infty}\{B(\lambda+\omega)+B(\lambda-\omega)-\omega\} e^{-x \omega} d \omega .
$$

Since $B^{\prime}(t)-1 / 2$ is an increasing, odd function,

$$
B(\lambda+\omega)-B(\lambda-\omega)-\omega=\int_{\lambda-\omega}^{\lambda+\omega}\left\{B^{\prime}(t)-1 / 2\right\} d t \geqslant \int_{-\omega}^{\omega}\left\{B^{\prime}(t)-1 / 2\right\} d t=0,
$$

for $\omega>0$. Therefore $M_{2}(\lambda, x) \geqslant e^{-\lambda|x|}$.

To prove the lower bound, we note that the right-hand side of (3.3) defines an analytic function for $\operatorname{Re}(z)>-1$; consequently (3.3) is true for $\operatorname{Re}(z)>-1$ by analytic continuation. If $x>0$, then $x-1 / 2>-1$, so

$$
m_{2}(\lambda, x)-e^{-\lambda x}=e^{-\lambda / 2}\left(\frac{\cos \pi x}{\pi}\right)^{2} \int_{0}^{\infty}\left\{B(\lambda-\omega) e^{\omega / 2}+B(\lambda+\omega) e^{-\omega / 2}\right\} e^{-x \omega} d \omega .
$$

Let $C(\omega)=B(\omega) e^{-\omega / 2}$, so that

$$
m_{2}(\lambda, x)-e^{-\lambda x}=\left(\frac{\cos \pi x}{\pi}\right)^{2} \int_{0}^{\infty}\{C(\lambda+\omega)-C(\lambda-\omega)\} e^{-x \omega} d \omega .
$$

$C(\omega)$ is an even function which is strictly decreasing for $\omega>0$. Therefore

$$
C(\lambda-\omega)=C(|\lambda-\omega|) \geqslant C(\lambda+\omega)
$$

if $\omega>0$. This proves the required lower bound.

With a more detailed calculation, we could show that

$$
M_{2}(\lambda, x)<e^{-\lambda|x|}+\left(\frac{\sin \pi x}{\pi x}\right)^{2}
$$

and

$$
m_{2}(\lambda, x) \geqslant \min \left\{e^{-\lambda / 2}, e^{-\lambda|x|}-\left(x^{2}-1 / 4\right)^{-1}\left(\frac{\cos \pi x}{\pi}\right)^{2}\right\} .
$$

Throughout the remainder of this section $\nu$ will denote a finite Borel measure on $(0, \infty)$ such that

$$
\int_{0}^{\infty} \lambda^{-1} d \nu(\lambda)<\infty .
$$


By Tonelli's theorem

$$
\int_{0}^{\infty} \int_{0}^{\infty} e^{-\lambda x} d \nu(\lambda) d x=\int_{0}^{\infty} \lambda^{-1} d \nu(\lambda)<\infty,
$$

and so each function $f(x)$ defined by (1.18), (1.19) or (1.20) is integrable on the real line. In each of these cases the periodic function defined by (1.9) is easily seen to have a unique maximum and unique minimum on $[-1 / 2,1 / 2)$. Thus for each such $f$ the functions $M_{f}$ and $m_{f}$ are uniquely determined.

THEOREM 9. If $f(x)$ is defined by either (1.18), (1.19) or (1.20), then $M_{f}$ is the unique extreme majorant of $f$, and $m_{f}$ is the unique extreme minorant of $f$.

Proof. The function $f$ satisfies (1.8) since

$$
\begin{aligned}
\sum_{n=-\infty}^{\infty}|f(x+n)| & \leqslant \int_{0}^{\infty} \sum_{n=-\infty}^{\infty} e^{-\lambda|x+n|} d \nu(\lambda) \\
& \leqslant 2 \int_{0}^{\infty}\left(e^{\lambda}-1\right)^{-1} e^{\lambda} d \nu(\lambda) \ll \int_{0}^{\infty}\left(\lambda^{-1}+1\right) d \nu(\lambda)
\end{aligned}
$$

uniformly in $x$, and if $|x| \leqslant 1 / 2$, then

$$
\begin{aligned}
\sum_{n \neq 0}\left|f^{\prime}(x+n)\right| \log 2|n| & \leqslant \int_{0}^{\infty} \lambda \sum_{n \neq 0} e^{-\lambda|x+n|} \log 2|n| d \nu(\lambda) \\
& \leqslant 2 \int_{0}^{\infty} \lambda e^{\lambda / 2} \sum_{n=1}^{\infty} e^{-\lambda n} \log 2 n d \nu(\lambda) \\
& \ll \int_{0}^{\infty} \lambda \sum_{n=1}^{\infty} e^{-\lambda n / 2} n d \nu(\lambda) \ll \int_{0}^{\infty} \lambda^{-1} d \nu(\lambda)
\end{aligned}
$$

Now suppose that $f(x)$ is defined by (1.18); then

$$
M_{f}(x)=\int_{0}^{\infty} M(\lambda, x) d \nu(\lambda)
$$

and

$$
\hat{M}_{f}(t)=\int_{0}^{\infty} \hat{M}(\lambda, t) d \nu(\lambda)=0
$$

if $|t| \geqslant 1$. By Theorem $6, M_{f}$ is a majorant of $f$; by Theorem $1, M_{f}$ is the unique extreme majorant.

The proofs for $m_{f}$ and for $f$ defined by (1.19) or (1.20) are essentially the same.

4. Tauberian theorems. Throughout this section we let $s=\sigma+i t=\sigma+2 \pi i \tau$ denote a complex variable and let $\alpha$ be a Borel measure on $[0, \infty)$. We also suppose that the Laplace-Stieltjes transform

$$
F(s)=\int_{0-}^{\infty} e^{-s x} d \alpha(x)
$$

defines an analytic function of $s$ in the open half plane $\sigma>r$ for some fixed $r>0$. One form of the classical Tauberian theorem of Wiener-Ikehara [1], [10] states that if for some constant $A>0$ the function $F(s)-A(s-r)^{-1}$ extends to a continuous function in the closed half plane $\sigma \geqslant r$, then

$$
\lim _{x \rightarrow \infty} e^{-r x} \alpha([0, x])=A r^{-1}
$$


By using the extremal functions $m(\lambda, x)$ and $M(\lambda, x)$ we obtain the following refinement of the Wiener-Ikehara theorem.

THEOREM 10. Let $T>0$ be fixed and suppose that $F(s)-A(s-r)^{-1}$ extends to a continuous function on the set

$$
\{s \in C: s=\sigma+2 \pi i \tau, \sigma>r\} \cup\{s \in C: s=r+2 \pi i \tau,|\tau|<T\} .
$$

Then

$$
\begin{aligned}
A T^{-1}\left\{\exp \left(r T^{-1}\right)-1\right\}^{-1} & \leqslant \liminf _{x \rightarrow \infty} e^{-r x} \alpha([0, x]) \\
& \leqslant \limsup _{x \rightarrow \infty} e^{-r x} \alpha([0, x]) \\
& \leqslant A T^{-1} \exp \left(r T^{-1}\right)\left\{\exp \left(r T^{-1}\right)-1\right\}^{-1} .
\end{aligned}
$$

Also, the upper and lower bounds on each side of (4.2) are best possible.

We deduce Theorem 10 from a general result in which $F(s)$ has a more complicated behavior near the line $\sigma=r$. To describe this situation we suppose that $\left\{u_{n}\right\}_{n=-N}^{N}$ and $\{a(n)\}_{n=-N}^{N}$ are complex numbers, $u_{n}=r+2 \pi i \xi_{n}, u_{-n}=\bar{u}_{n}$, and $a(-n)=\overline{a(n)}$. We then define

$$
G(s)=\sum_{n=-N}^{N} a(n)\left\{s-u_{n}\right\}^{-1}
$$

THEOREM 11. Let $T>0$ be fixed and suppose that

$$
H_{\sigma}(\tau)=F(s)-G(s)
$$

is Cauchy in $L^{1}([-T, T])$ norm as $\sigma \rightarrow r+$ for some fixed $r>0$. For $x>0$,

$$
\begin{array}{r}
T^{-1} \sum_{n=-N}^{N} a(n) \hat{m}\left(r T^{-1}, \xi_{n} T^{-1}\right) e\left(\xi_{n} x\right)+o_{T}(1)<e^{-r x} \alpha([0, x]) \\
\leqslant T^{-1} \sum_{n=-N}^{N} a(n) \hat{M}\left(r T^{-1}, \xi_{n} T^{-1}\right) e\left(\xi_{n} x\right)+o_{T}(1),
\end{array}
$$

where $o_{T}(1)$ denotes a function of $T$ and $x$ which tends to zero as $x \rightarrow \infty$ for each fixed $T$.

If we define

$$
J(\lambda, x)=\frac{1}{2}\{m(\lambda, x)+M(\lambda, x)\}
$$

and use the identity (3.7), then (4.3) can be written in the equivalent form

$$
\begin{aligned}
\mid e^{-r x} \alpha([0, x])- & T^{-1} \sum_{n=-N}^{N} a(n) \hat{J}\left(r T^{-1}, \xi_{n} T^{-1}\right) e\left(\xi_{n} x\right) \mid \\
& \leqslant(2 T)^{-1} \sum_{n=-N}^{N} a(n)\left(1-\left|\xi_{n}\right| T^{-1}\right)^{+} e\left(\xi_{n} x\right)+o_{T}(1) .
\end{aligned}
$$

Here we use $y^{+}=\max \{y, 0\}$. It is easy to check that the various trigonometric polynomials in (4.3) and (4.4) are all real valued functions of $x$ because of our 
hypotheses on $a(n)$ and $\xi_{n}$. We also remark that Theorem 11 is an analogue of a Tauberian theorem proved by Diamond [2].

Proof of Theorem 11. We prove only the upper bound in (4.3); the proof of the lower bound is very similar. For all real $x$ and $\lambda>0$,

$$
E(\lambda, x)=E\left(\lambda T^{-1}, x T\right)<M\left(\lambda T^{-1}, x T\right)
$$

and

$$
\int_{-\infty}^{\infty} M\left(\lambda T^{-1}, x T\right) e(-\tau x) d x=T^{-1} \hat{M}\left(\lambda T^{-1}, \tau T^{-1}\right) .
$$

Thus the right-hand side of (4.5) is zero if $|\tau| \geq T$. Now if $\sigma>r$ and $x>0$ then

$$
\begin{aligned}
e^{-\sigma x} \alpha([0, x]) & =\int_{0}^{x+} e^{-\sigma(x-y)-\sigma y} d \alpha(y) \\
& =\int_{-\infty}^{\infty} E(\sigma, x-y) E(\sigma, y) d \alpha(y) \\
& \leqslant \int_{-\infty}^{\infty} M\left(\sigma T^{-1},(x-y) T\right) E(\sigma, y) d \alpha(y) .
\end{aligned}
$$

The right-hand side of (4.6) is easily seen to be an integrable function of $x$ by Tonelli's theorem. Hence

$$
\begin{aligned}
\int_{-\infty}^{\infty} \int_{-\infty}^{\infty} M\left(\sigma T^{-1}\right. & ,(x-y) T) E(\sigma, y) d \alpha(y) e(-\tau x) d x \\
= & \int_{-\infty}^{\infty} E(\sigma, y) T^{-1} \hat{M}\left(\sigma T^{-1}, \tau T^{-1}\right) e(-\tau y) d \alpha(y) \\
& =T^{-1} \hat{M}\left(\sigma T^{-1}, \tau T^{-1}\right) F(\sigma+2 \pi i \tau)
\end{aligned}
$$

by Fubini's theorem. Since $\hat{M}\left(\sigma T^{-1}, \tau T^{-1}\right)$ has compact support the Fourier inversion formula applies, and

$$
\begin{aligned}
\int_{-\infty}^{\infty} T^{-1} \hat{M}\left(\sigma T^{-1}, \tau T^{-1}\right) F(\sigma+2 \pi i \tau) e(\tau x) d \tau \\
=\int_{-\infty}^{\infty} M\left(\sigma T^{-1},(x-y) T\right) E(\sigma, y) d \alpha(y)
\end{aligned}
$$

Next we observe that

$$
\begin{aligned}
\int_{-\infty}^{\infty} E(\sigma, y) & \left\{\sum_{n=-N}^{N} a(n) e^{u_{n} y}\right\} e(-\tau y) d y \\
= & \sum_{n=-N}^{N} a(n) \int_{0}^{\infty} e^{-\left(s-u_{n}\right) y} d y=G(s)
\end{aligned}
$$

for $\operatorname{Re}(s)=\sigma>r$. It follows that

$$
\begin{aligned}
\int_{-\infty}^{\infty} T^{-1} \hat{M}\left(\sigma T^{-1}, \tau T^{-1}\right) G(\sigma+2 \pi i \tau) e(\tau x) d \tau \\
\quad=\int_{-\infty}^{\infty} M\left(\sigma T^{-1},(x-y) T\right) E(\sigma, y)\left\{\sum_{n=-N}^{N} a(n) e^{u_{n} y}\right\} d y \\
\quad=\int_{-\infty}^{\infty} M\left(\sigma T^{-1},(x-y) T\right) E(\sigma-r, y)\left\{\sum_{n=-N}^{N} a(n) e\left(\xi_{n} y\right)\right\} d y .
\end{aligned}
$$


Combining (4.8), (4.9) and (4.10), we find that for $\sigma>r$ and $x>0$,

$$
\begin{aligned}
e^{-\sigma x} \alpha([0, x]) \leqslant & \int_{-\infty}^{\infty} T^{-1} \hat{M}\left(\sigma T^{-1}, \tau T^{-1}\right) F(\sigma+2 \pi i \tau) e(\tau x) d \tau \\
= & \int_{-\infty}^{\infty} T^{-1} \hat{M}\left(\sigma T^{-1}, \tau T^{-1}\right) H_{\sigma}(\tau) e(\tau x) d \tau \\
& +\int_{-\infty}^{\infty} M\left(\sigma T^{-1},(x-y) T\right) E(\sigma-r, y)\left\{\sum_{n=-N}^{N} a(n) e\left(\xi_{n} y\right)\right\} d y .
\end{aligned}
$$

By the completeness of $L^{1}([-T, T])$ and the fact that $\hat{M}\left(\sigma T^{-1}, \tau T^{-1}\right)$ is zero if $|\tau| \geqslant T$,

$$
\begin{aligned}
\lim _{\sigma \rightarrow r+} \int_{-\infty}^{\infty} & T^{-1} \hat{M}\left(\sigma T^{-1}, \tau T^{-1}\right) H_{\sigma}(\tau) e(\tau x) d \tau \\
& =\int_{-T}^{T} T^{-1} \hat{M}\left(r T^{-1}, \tau T^{-1}\right) H_{r}(\tau) e(\tau x) d \tau,
\end{aligned}
$$

where $H_{r}(\tau)$ is integrable on [-T,T]. Using the Riemann-Lebesgue lemma, we see that the right-hand side of $(4.12)$ is $o_{T}(1)$ as $x \rightarrow \infty$. The other integral on the right of $(4.11)$ is

$$
\begin{gathered}
\lim _{\sigma \rightarrow r+} \int_{-\infty}^{\infty} M\left(\sigma T^{-1},(x-y) T\right) E(\sigma-r, y)\left\{\sum_{n=-N}^{N} a(n) e\left(\xi_{n} y\right)\right\} d y \\
=\int_{0}^{\infty} M\left(r T^{-1},(x-y) T\right)\left\{\sum_{n=-N}^{N} a(n) e\left(\xi_{n} y\right)\right\} d y
\end{gathered}
$$

by Theorem 6 and the dominated convergence theorem. The right-hand side of (4.13) is

$$
\begin{aligned}
\int_{-\infty}^{x} M\left(r T^{-1}, \omega T\right)\left\{\sum_{n=-N}^{N} a(n) e\left(\xi_{n} x-\xi_{n} \omega\right)\right\} d \omega \\
=\sum_{n=-N}^{N} a(n)\left\{\int_{-\infty}^{\infty} M\left(r T^{-1}, \omega T\right) e\left(-\xi_{n} \omega\right) d \omega\right\} e\left(\xi_{n} x\right) \\
-\int_{x}^{\infty} M\left(r T^{-1}, \omega T\right)\left\{\sum_{n=-N}^{N} a(n) e\left(\xi_{n} x-\xi_{n} \omega\right)\right\} d \omega \\
=\sum_{n=-N}^{N} a(n) T^{-1} \hat{M}\left(r T^{-1}, \xi_{n} T^{-1}\right) e\left(\xi_{n} x\right)+o_{T}(1)
\end{aligned}
$$

as $x \rightarrow \infty$. Finally we use the inequality (4.11) together with the estimates (4.12), (4.13) and (4.14) to obtain the upper bound in (4.3). This completes our proof.

Proof of Theorem 10. We apply Theorem 11 with $N=0, \xi_{0}=0$ and $a(0)=A$. Since $F(s)-A(s-r)^{-1}$ extends to a continuous function on the set (4.1) it is Cauchy in $L^{1}([-T+\varepsilon, T-\varepsilon])$ norm for every $\varepsilon, 0<\varepsilon<T$. Using (4.3) we have

$$
\limsup _{x \rightarrow \infty} e^{-r x} \alpha([0, x]) \leqslant A(T-\varepsilon)^{-1} \hat{M}\left(r(T-\varepsilon)^{-1}, 0\right) \text {. }
$$

If we apply the identity (2.6) with $t=0$ and let $\varepsilon \rightarrow 0+$ in (4.15) we obtain the upper bound in (4.2). The lower bound is proved in a similar way. To show that 
these bounds are best possible let $\alpha$ be the measure with point mass $A T^{-1} \exp \left(r n T^{-1}\right)$ at $n T^{-1}$ for $n=1,2,3, \cdots$. Then

$$
\begin{aligned}
F(s) & =\int_{0-}^{\infty} e^{-s x} d \alpha(x)=A T^{-1} \sum_{n=1}^{\infty} \exp \left\{(r-s) n T^{-1}\right\} \\
& =A T^{-1}\left(\exp \left\{(s-r) T^{-1}\right\}-1\right)^{-1}
\end{aligned}
$$

if $\sigma>r$. It follows that $F(s)-A(s-r)^{-1}$ is analytic for $\sigma>r$ and at each point of the line $\sigma=r,|\tau|<T$. We also have

$$
\begin{aligned}
e^{-r x} \alpha([0, x])= & e^{-r x} A T^{-1} \sum_{1<n<x T} \exp \left\{r n T^{-1}\right\} \\
= & A T^{-1} \exp \left(r T^{-1}\right)\left\{\exp \left(r T^{-1}\right)-1\right\}^{-1} \\
& \cdot \exp \left\{-r T^{-1}(T x-[T x])\right\}+o_{T}(1)
\end{aligned}
$$

as $x \rightarrow \infty$. Since

$$
0=\liminf _{x \rightarrow \infty} T x-[T x], \quad 1=\limsup _{x \rightarrow \infty} T x-[T x],
$$

we obtain the same bounds given in (4.2).

Note ADDED IN PROOF. A result similar to Theorem 10 was proved by Heilbronn and Landau, Math. Z. 37 (1933), pp. 10-16 and pp. 18-21. Their result is weaker than ours in that the upper and lower bounds in (4.2) are unspecified functions of $A, r$ and $T$. A further discussion of such Tauberian theorems can be found in Ingham's paper, Proc. London Math. Soc. (2) 38 (1935), pp. 458-480. We wish to thank Professor P. T. Bateman for calling our attention to these papers.

5. More applications. Selberg [8] has observed that several standard applications of the large sieve may be done via majorizing functions. In this section, we will supply the details to Selberg's observation, and we will prove these results in a more general setting.

We assume that $f$ and $F$ are bounded functions in $L^{1}(\mathbf{R})$ which satisfy

$$
\begin{gathered}
f(x) \leqslant F(x) \text { for all } x, \text { and } \\
\hat{F}(y)=0 \text { for }|y|>\delta
\end{gathered}
$$

for some $\delta>0$. We also need some condition which allows us to apply the Poisson summation formula to $F(x)$; e.g.

$F$ is of bounded total variation on $\mathbf{R}$.

Note that if $f$ satisfies (1.5)-(1.8) and $M_{f}$ is defined by (1.13), then

$$
\begin{aligned}
\int_{-\infty}^{\infty}\left|M_{f}^{\prime}(x)\right| d x \ll & \sum_{n}|f(n)| \int_{-\infty}^{\infty} \frac{|\sin \pi x|}{x^{2}} \frac{|\pi x \cos \pi x-\sin \pi x|}{|x|} d x \\
& +\sum_{n \neq 0}\left|f^{\prime}(n)\right| \int_{-\infty}^{\infty}\left|\frac{\sin \pi x}{x}-\frac{\sin \pi x}{x-n}\right| d x \\
& \ll \sum_{n}|f(n)|+\sum_{n \neq 0}\left|f^{\prime}(n)\right| \log 2|n|<\infty .
\end{aligned}
$$

Thus $M_{f}$ has bounded total variation on $\mathbf{R}$. 
If $f(x)=E(\lambda, x)$, then $F(x)=M\left(\lambda \delta^{-1}, \delta x\right)$ satisfies (5.1) through (5.3), and

$$
\hat{F}(0)=e^{\lambda / \delta} / \delta\left(e^{\lambda / \delta}-1\right) \text {. }
$$

If $f=\chi_{[M+1, M+N]}$, we may take $l=(N-1) \delta$ in Selberg's construction (1.4), $F(x)=G_{l}(\delta(x-M-1))$, and

$$
\hat{F}(0)=N+\delta^{-1}-1 .
$$

Most of our applications are based on

LemMa 12. Suppose F satisfies (5.3) and (5.2) with $\delta<q^{-1}$. Then

$$
\sum_{n \equiv a \bmod q} F(n)=\hat{F}(0) / q
$$

for any integer a. Moreover, if $\chi$ is a Dirichlet character $\bmod q$, then

$$
\sum_{n} F(n) \chi(n)= \begin{cases}(\varphi(q) / q) \hat{F}(0) & \text { if } \chi \text { is principal, } \\ 0 & \text { otherwise. }\end{cases}
$$

Proof. Let $G(n)=F(q n+a)$, so that

$$
\hat{G}(y)=(1 / q) e(a y / q) \hat{F}(y / q) .
$$

By the Poisson summation formula,

$$
\sum_{n \equiv a \bmod q} F(n)=\sum_{n} G(n)=\sum_{n} \hat{G}(n)=(1 / q) \hat{F}(0)
$$

This proves the first assertion. The second assertion follows upon noting that

$$
\sum_{n} F(n) \chi(n)=\sum_{a=1}^{q} \chi(a) \sum_{n=a \bmod q} F(n)=(\hat{F}(0) / q) \sum_{a=1}^{q} \chi(a) .
$$

Our first application is to the large sieve.

THEOREM 13. Suppose $G(n)$ is a polynomial with integral coefficients, $\mathscr{P}$ is a set of primes, and $P(z)=\Pi_{p<z, p \in \mathcal{P}} p$. Define $\omega(d)$ to be the number of solutions of $G(r) \equiv 0 \bmod d$. Suppose $f$ and $F$ satisfy (5.1), (5.2), and (5.3) with $\delta=z^{-2}$. Then

$$
\sum_{\substack{n \\(G(n), P(z))=1}} f(n) \leqslant \hat{F}(0) V^{-1}
$$

where

$$
V=\sum_{r<z} \mu^{2}(r) \prod_{p \mid r}\left(\frac{\omega(p)}{p-\omega(p)}\right) .
$$

Proof. If $d \mid P(z)$, define

$$
\lambda_{d}=\frac{\mu(d) d}{V \prod_{p \mid d}(p-\omega(p))} \sum_{\substack{r<z / d \\(r, d)=1}} \mu^{2}(r) \prod_{p \mid r}\left(\frac{\omega(p)}{p-\omega(p)}\right)
$$


if $d \nmid P(z)$, define $\lambda_{d}=0$. Since $\lambda_{1}=1$,

$$
\begin{aligned}
\sum_{(G(n), P(z))=1} f(n) & \leqslant \sum_{n} F(n)\left(\sum_{d \mid G(n)} \lambda_{d}\right)^{2} \\
& =\sum_{d, e} \lambda_{d} \lambda_{e} \sum_{\substack{n \\
G(n) \equiv 0 \bmod [d, e]}} F(n) \\
& =\hat{F}(0) \sum_{d, e}\left(\lambda_{d} \lambda_{e} /[d, e]\right) \omega([d, e]),
\end{aligned}
$$

where we have used Lemma 12 and the definition of $\omega$ in the last line. Since the sum in the last line is $V^{-1}$ [3, Chapter 3], this completes the proof.

By Theorem 13 and (5.5),

$$
\sum_{\substack{n=M+1 \\(G(n), P(z))=1}}^{M+N} 1 \leqslant \frac{N-1+z^{2}}{V} .
$$

This is the classical large sieve, and the proof given here was first given by Selberg [8]. In another paper [9], Selberg considered a more general situation in which one sieves by prime powers.

We mention one other application. Let $G(n)=q n+a$, where $(a, q)=1$. Here $\omega(d)=1$ if $(d, q)=1$ and 0 otherwise. Furthermore [3, Theorem 3.3], $V>$ $(\varphi(q) / q) \log z$. We now apply Theorem 13 with $f(n)=E(1 / Y, n)$ and $z=$ $Y^{1 / 2}(\log Y)^{-1}$ to obtain

$$
\sum_{\substack{n=0 \\ q n+a \text { prime }}}^{\infty} e^{-n / Y} \leqslant \frac{z^{2}\left(e^{z^{2} / Y}\right)}{V\left(e^{z^{2} / Y}-1\right)}=\frac{2 q Y}{\varphi(q) \log Y}\left\{1+O\left(\frac{\log \log Y}{\log Y}\right)\right\} .
$$

This may be regarded as a weighted form of the Brun-Titchmarsh theorem.

Our next application is to character sum inequalities. Here we will use $\Sigma_{x \bmod q}$ to denote a sum over all characters $\bmod q$ and $\Sigma_{x \bmod q}^{*}$ to denote a sum over all primitive characters $\bmod q$.

THeOREM 14. Suppose $f$ and $F$ satisfy (5.1), (5.2) and (5.3) with $\delta=Q^{-2}$, and $f(x) \geqslant 0$ for all $x$. Then

$$
\sum_{q<Q} \frac{q}{\varphi(q)} \sum_{\chi \bmod q}^{*}\left|\sum_{n} f^{1 / 2}(n) a(n) \chi(n)\right|^{2} \leqslant \hat{F}(0) \sum_{n}|a(n)|^{2} .
$$

Proof. By duality, it suffices to show that

$$
\sum_{n} f(n)\left|\sum_{q<Q} \sum_{\chi \bmod q}^{*} b(\chi) \chi(n)\right|^{2}<\hat{F}(0) \sum_{q<Q} \frac{\varphi(q)}{q} \sum_{\chi \bmod q}^{*}|b(\chi)|^{2} .
$$

The left-hand side of (5.7) is

$$
\begin{aligned}
& \leqslant \sum_{n} F(n)|\cdots|^{2} \\
& =\sum_{q_{1}<Q} \sum_{q_{2}<Q} \sum_{\chi_{1} \bmod q_{1}}^{*} \sum_{\chi_{2} \bmod q_{2}}^{*} b\left(\chi_{1}\right) \overline{b\left(\chi_{2}\right)} \sum_{n} F(n) \chi_{1}(n) \overline{\chi_{2}(n)} .
\end{aligned}
$$


By Lemma 12, the inner sum is 0 unless $q_{1}=q_{2}=q$ and $\chi_{1}=\chi_{2}$, when the inner sum is $(\varphi(q) / q) \hat{F}(0)$. This proves (5.7), so (5.6) follows.

In the case $f=\chi_{[M+1, M+N]}$, the inequality (5.7) is well known (cf. [4, Theorem 2.5]). The proof given here is implicit in Selberg [8]. Note that the only property of primitive characters used in the proof of Theorem 14 was that $\chi_{1} \bar{\chi}_{2}$ is principal if and only if $\chi_{1}=\chi_{2}$. Thus we have proved the following more general result.

THEOREM 15. Let 2 be any set of Dirichlet characters such that if $\chi_{1}, \chi_{2} \in \mathcal{2}$ and $\chi_{1} \bar{\chi}_{2}$ is principal, then $\chi_{1}=\chi_{2}$. For $\chi \in \mathcal{Q}$, let $q(\chi)$ denote the modulus of $\chi$, and set

$$
Q=\max _{x_{1}, x_{2} \in \mathcal{Q}}\left[q\left(x_{1}\right), q\left(x_{2}\right)\right]
$$

If $f$ and $F$ satisfy (5.1), (5.2), and (5.3) with $\delta=Q^{-1}$, and $f(x)>0$ for all $x$, then

$$
\sum_{\chi \in \mathcal{L}} \frac{q(\chi)}{\varphi(q(\chi))}\left|\sum_{n} f^{1 / 2}(n) a(n) \chi(n)\right|^{2} \leqslant \hat{F}(0) \sum_{n}|a(n)|^{2} .
$$

In particular, if $f$ and $F$ satisfy (5.1), (5.2) and (5.3) with $\delta=q^{-1}$, then

$$
\sum_{\chi \bmod q}\left|\sum_{n} f^{1 / 2}(n) a(n) \chi(n)\right|^{2} \leqslant \frac{\varphi(q)}{q} \hat{F}(0) \sum_{n}|a(n)|^{2}
$$

(cf. Theorem 6.2 of Montgomery [4]) and

$$
\sum_{d \mid q} \frac{d}{\varphi(d)} \sum_{\chi \bmod d}^{*}\left|\sum_{n} f^{1 / 2}(n) a(n) \chi(n)\right|^{2} \leqslant \hat{F}(0) \sum_{n}|a(n)|^{2}
$$

(cf. Theorem 6.3 of Montgomery [4]). Furthermore, the proofs of Theorems 13 and 14 may be combined. Thus if $f$ and $F$ satisfy (5.1), (5.2) and (5.3) with $\delta=Q^{-2} R^{-2}$, then

$$
\sum_{q<Q} \sum_{\chi \bmod q}^{*}\left|\sum_{n} f^{1 / 2}(n) a(n) \chi(n) \sum_{d \mid n} \lambda_{d}\right|^{2} \leqslant \hat{F}(0) \sum_{n}|a(n)|^{2} \sum_{d, e} \frac{\lambda_{d} \lambda_{e}}{[d, e]} .
$$

Inequalities of this sort have been used to investigate the distribution of primes in arithmetic progressions [7], [8].

For our final application, we require both minorizing and majorizing functions. We assume that $f, F_{+}$, and $F_{-}$are integrable functions such that

$$
\begin{aligned}
& F_{-}(x) \leqslant f(x) \leqslant F_{+}(x) \text { for all real } x, \text { and } \\
& \hat{F}_{-}(t)=\hat{F}_{+}(t)=0 \text { for }|t| \geqslant \delta .
\end{aligned}
$$

It can be shown that

$$
\chi_{[0, l]}(x) \geqslant g_{l}(x)=G_{l}(x)-\left(\frac{\sin \pi x}{\pi x}\right)^{2}-\left(\frac{\sin \pi(l-x)}{\pi(l-x)}\right)^{2},
$$

where $G_{l}$ is Selberg's function defined in (1.4). Therefore if $f=\chi_{[M+1, M+N]}$, we may take $F_{-}(x)=g_{(N-1) \delta}(\delta(x-M-1))$ and $\hat{F}_{-}(0)=N-\delta^{-1}-1$. If $f(x)=$ $E(\lambda, x)$, we may take $F_{-}(x)=m\left(\lambda \delta^{-1}, \delta x\right)$ and

$$
\hat{F}_{-}(0)=1 / \delta\left(e^{\lambda / \delta}-1\right) \text {. }
$$


THEOREM 16. Suppose that $f, F_{+}$, and $F_{-}$satisfy (5.8) and (5.9), and that $\left\{\xi_{n}\right\}$ is a sequence of real numbers such that $\left|\xi_{n}-\xi_{m}\right| \geqslant \delta$ for $n \neq m$. Then

$$
\hat{F}_{-}(0) \sum_{n}|a(n)|^{2} \leqslant \int_{-\infty}^{\infty} f(t)\left|\sum_{n} a(n) e\left(t \xi_{n}\right)\right|^{2} d t<\hat{F}_{+}(0) \sum_{n}|a(n)|^{2} .
$$

Proof. To prove the upper bound, we observe that

$$
\begin{aligned}
\int_{-\infty}^{\infty} f(t)\left|\sum_{n} a(n) e\left(t \xi_{n}\right)\right|^{2} d t & \leqslant \int_{-\infty}^{\infty} F_{+}(t)\left|\sum_{n}\right|^{2} d t \\
& =\sum_{m} \sum_{n} a(m) \overline{a(n)} \hat{F}_{+}\left(\xi_{n}-\xi_{m}\right)=\hat{F}_{+}(0) \sum_{n}|a(n)|^{2} .
\end{aligned}
$$

The lower bound is proved in the same fashion.

Note that

$$
\int_{-\infty}^{\infty} f(t)\left|\sum_{n} a(n) e\left(t \xi_{n}\right)\right|^{2} d t=\sum_{m} \sum_{n} a(m) \overline{a(n)} \hat{f}\left(\xi_{m}-\xi_{n}\right) .
$$

Therefore Theorem 16 gives a bound for such bilinear forms. In particular, if $f(t)=E(\lambda, t)$, then

$$
\hat{f}(y)=1 /(\lambda+2 \pi i y)
$$

consequently,

$$
\frac{1}{\delta\left(e^{\lambda / \delta}-1\right)} \sum_{n}|a(n)|^{2} \leqslant \sum_{m} \sum_{n} \frac{a(m) \overline{a(n)}}{\lambda+2 \pi i\left(\xi_{m}-\xi_{n}\right)}<\frac{e^{\lambda / \delta}}{\delta\left(e^{\lambda / \delta}-1\right)} \sum_{n}|a(n)|^{2} .
$$

If we subtract off the diagonal terms and let $\lambda \rightarrow 0$, we obtain

$$
\left|\sum_{m \neq n} \frac{a(m) \overline{a(n)}}{\xi_{m}-\xi_{n}}\right| \leqslant \pi \delta^{-1} \sum_{n}|a(n)|^{2} .
$$

This generalization of Hilbert's inequality was first proved by Montgomery and Vaughan [6]. It can be shown that (5.11) and (5.12) are best possible.

The integration over $t$ in Theorem 16 may be replaced by a summation over integers. In this manner one can show that if $f, F_{-}$, and $F_{+}$satisfy (5.9) and (5.10), and $F_{-}$and $F_{+}$are of bounded variation on $\mathbf{R}$, then

$$
\hat{F}_{-}(0) \sum_{n}|a(n)|^{2} \leqslant \sum_{r=-\infty}^{\infty} f(r)\left|\sum_{n} a(n) e\left(r \xi_{n}\right)\right|^{2}<\hat{F}_{+}(0) \sum_{n}|a(n)|^{2}
$$

provided

$$
\left\|\xi_{n}-\xi_{m}\right\|=\min _{q \in \mathbf{Z}}\left|\xi_{n}-\xi_{m}-q\right|>\delta
$$

for $n \neq m$. In particular, if (5.13) is true, then

$$
\begin{aligned}
\frac{e^{\lambda / 2}}{\delta\left(e^{\lambda / \delta}-1\right)} \sum_{n}|a(n)|^{2} & \leqslant \sum_{m} \sum_{n} \frac{a(m) \overline{a(n)}}{\sinh \left(\lambda / 2+\pi i\left(\xi_{n}-\xi_{m}\right)\right)} \\
& \leqslant \frac{e^{\lambda / 2} e^{\lambda / \delta}}{\delta\left(e^{\lambda / \delta}-1\right)} \sum_{n}|a(n)|^{2} .
\end{aligned}
$$


If we let $\lambda \rightarrow 0$, we obtain

$$
\left|\sum_{\substack{m, n \\ m \neq n}} \frac{a(m) \overline{a(n)}}{\sin \pi\left(\xi_{m}-\xi_{n}\right)}\right| \leqslant \delta^{-1} \sum_{n}|a(n)|^{2} ;
$$

this is another result of Montgomery and Vaughan [6].

We close by mentioning some open questions on bilinear forms. Let $z_{1}, z_{2}, \ldots$ be complex numbers with nonnegative real part. What is the best possible bound for

$$
\sum_{m \neq n} \frac{a(n) \overline{a(m)}}{z_{n}+\bar{z}_{m}} ?
$$

A bound for (5.14) will have to take into account the spacing of the $z_{n}$ 's, but it is not clear what the best measure of spacing is. Note that (5.14) reduces to (5.11) when $0<\lambda / 2=\operatorname{Re} z_{1}=\operatorname{Re} z_{2}=\ldots$, and it reduces to (5.12) when $0=\operatorname{Re} z_{1}=$ $\operatorname{Re} z_{2}=\ldots$

Another open question arises from the weighted version of Hilbert's inequality. Montgomery and Vaughan [6] have shown that if $\left|\xi_{m}-\xi_{n}\right|>\delta_{m}$ for any $n$ distinct from $m$, then

$$
\left|\sum_{m \neq n} \frac{a(m) \overline{a(n)}}{\xi_{m}-\xi_{n}}\right| \leqslant \frac{3}{2} \pi \sum_{n}|a(n)|^{2} \delta_{n}^{-1} .
$$

Is it possible to prove (5.15) by means of majorizing functions? The questions in (5.14) and (5.15) are in some sense analogous. It would also be desirable to have a weighted bound in (5.14).

\section{REFERENCES}

1. K. Chandresekharan, Introduction to analytic number theory, Springer, Berlin, 1968.

2. H. G. Diamond, Changes of sign of $\pi(x)-l i(x)$, Enseignement Math., Tome XXI, Fasc. 1, 1975.

3. H. Halberstam and H.-E. Richert, Sieve methods, Academic Press, London, 1974.

4. H. L. Montgomery, Topics in multiplicative number theory, Lecture Notes in Math., vol. 227, Springer, Berlin, 1971.

5. __ Analytic principle of the large sieve, Bull. Amer. Math. Soc. 84 (1978), 547-567.

6. H. L. Montgomery and R. C. Vaughan, Hilbert's inequality, J. London Math. Soc. (2) 8 (1974), 73-81.

7. Y. Motohashi, Primes in arithmetic progressions, Invent. Math. 44 (1978), 163-178.

8. A. Selberg, Remarks on sieves, Proc. 1972 Number Theory Conf., University of Colorado, Boulder, 1972, pp. 205-216.

9. ___ Multiplicative functions, Number Theory Day 1976, Lecture Notes in Math., vol. 626, Springer, Berlin, 1977, pp. 232-241.

10. N. Wiener, The Fourier integral and certain of its applications, Cambridge Univ. Press, 1933.

Department of Mathematics, University of TeXas, Austin, TeXas 78712 